\title{
IceCube sensitivity for low-energy neutrinos from nearby supernovae (Corrigendum)
}

\author{
IceCube Collaboration: R. Abbasi ${ }^{1}$, Y. Abdou ${ }^{2}$, T. Abu-Zayyad ${ }^{3}$, M. Ackermann ${ }^{10}$, J. Adams ${ }^{4}$, J. A. Aguilar ${ }^{1}$,
}

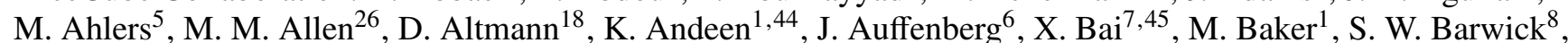
V. Baum ${ }^{33}$, R. Bay ${ }^{9}$, J. L. Bazo Alba ${ }^{10}$, K. Beattie ${ }^{11}$, J. J. Beatty ${ }^{12,13}$, S. Bechet ${ }^{14}$, J. K. Becker ${ }^{15}$, K.-H. Becker ${ }^{6}$, M. L. Benabderrahmane ${ }^{10}, \mathrm{~S}$. BenZvi $^{1}$, J. Berdermann ${ }^{10}$, P. Berghaus ${ }^{1}$, D. Berley ${ }^{16}$, E. Bernardini ${ }^{10}$, D. Bertrand ${ }^{14}$, D. Z. Besson ${ }^{17}$, D. Bindig ${ }^{6}$, M. Bissok ${ }^{18}$, E. Blaufuss ${ }^{16}$, J. Blumenthal ${ }^{18}$, D. J. Boersma ${ }^{18}$, C. Bohm ${ }^{19}$, D. Bose ${ }^{20}$, S. Böser ${ }^{21}$, O. Botner ${ }^{22}$, A. M. Brown ${ }^{4}$, S. Buitink ${ }^{11}$, K. S. Caballero-Mora ${ }^{26}$, M. Carson ${ }^{2}$, D. Chirkin ${ }^{1}$, B. Christy ${ }^{16}$, F. Clevermann ${ }^{23}$, S. Cohen ${ }^{24}$, C. Colnard ${ }^{25}$, D. F. Cowen ${ }^{26,27}$, A. H. Cruz Silva ${ }^{10}$, M. V. D' Agostino ${ }^{9}$, M. Danninger ${ }^{19}$, J. Daughhetee ${ }^{28}$, J. C. Davis ${ }^{12}$, C. De Clercq ${ }^{20}$, T. Degner ${ }^{21}$, L. Demirörs ${ }^{24}$, F. Descamps ${ }^{2}$, P. Desiati ${ }^{1}$, G. de Vries-Uiterweerd ${ }^{2}$, T. DeYoung ${ }^{26}$, J. C. Díaz-Vélez ${ }^{1}$, M. Dierckxsens ${ }^{14}$, J. Dreyer ${ }^{15}$, J. P. Dumm ${ }^{1}$, M. Dunkman ${ }^{26}$, J. Eisch $^{1}$, R. W. Ellsworth ${ }^{16}$, O. Engdegård ${ }^{22}$, S. Euler ${ }^{18}$, P. A. Evenson ${ }^{7}$, O. Fadiran ${ }^{29}$, A. R. Fazely ${ }^{30}$, A. Fedynitch ${ }^{15}$, J. Feintzeig ${ }^{1}$, T. Feusels ${ }^{2}$, K. Filimonov ${ }^{9}$, C. Finley ${ }^{19}$, T. Fischer-Wasels ${ }^{6}$, B. D. Fox ${ }^{26}$,

A. Franckowiak ${ }^{21}$, R. Franke ${ }^{10}$, T. K. Gaisser ${ }^{7}$, J. Gallagher ${ }^{31}$, L. Gerhardt ${ }^{11,9}$, L. Gladstone ${ }^{1}$, T. Glüsenkamp ${ }^{18}$, A. Goldschmidt ${ }^{11}$, J. A. Goodman ${ }^{16}$, D. Góra ${ }^{10}$, D. Grant ${ }^{32}$, T. Griesel ${ }^{33}$, A. Groß ${ }^{4,25}$, S. Grullon ${ }^{1}$, M. Gurtner ${ }^{6}$,

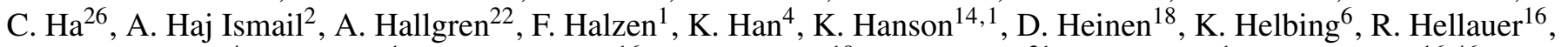
S. Hickford ${ }^{4}$, G. C. Hill ${ }^{1}$, K. D. Hoffman ${ }^{16}$, B. Hoffmann ${ }^{18}$, A. Homeier ${ }^{21}$, K. Hoshina ${ }^{1}$, W. Huelsnitz ${ }^{16,46}$, J.-P. Hülß ${ }^{18}$, P. O. Hulth ${ }^{19}$, K. Hultqvist ${ }^{19}$, S. Hussain ${ }^{7}$, A. Ishihara ${ }^{35}$, E. Jakobi ${ }^{10}$, J. Jacobsen ${ }^{1}$, G. S. Japaridze ${ }^{29}$, H. Johansson ${ }^{19}$, K.-H. Kampert ${ }^{6}$, A. Kappes ${ }^{36}$, T. Karg ${ }^{6}$, A. Karle ${ }^{1}$, P. Kenny ${ }^{17}$, J. Kiryluk ${ }^{11,9}$, F. Kislat ${ }^{10}$, S. R. Klein ${ }^{11,9}$, H. Köhne ${ }^{23}$, G. Kohnen ${ }^{34}$, H. Kolanoski ${ }^{36}$, L. Köpke ${ }^{33}$, S. Kopper ${ }^{6}$, D. J. Koskinen ${ }^{26}$, M. Kowalski ${ }^{21}$, T. Kowarik ${ }^{33}$, M. Krasberg ${ }^{1}$, G. Kroll ${ }^{33}$, N. Kurahashi ${ }^{1}$, T. Kuwabara ${ }^{7}$, M. Labare ${ }^{20}$, K. Laihem ${ }^{18}$, H. Landsman ${ }^{1}$, M. J. Larson ${ }^{26}$, R. Lauer ${ }^{10}$, J. Lünemann ${ }^{33}$, J. Madsen ${ }^{3}$, A. Marotta ${ }^{14}$, R. Maruyama ${ }^{1}$, K. Mase ${ }^{35}$, H. S. Matis ${ }^{11}$, K. Meagher ${ }^{16}$, M. Merck ${ }^{1}$, P. Mészáros ${ }^{27,26}$, T. Meures ${ }^{18}$, S. Miarecki ${ }^{11,9}$, E. Middell ${ }^{10}$, N. Milke ${ }^{23}$, J. Miller ${ }^{22}$, T. Montaruli ${ }^{1,37}$, R. Morse ${ }^{1}$, S. M. Movit ${ }^{27}$, R. Nahnhauer ${ }^{10}$, J. W. Nam ${ }^{8}$, U. Naumann ${ }^{6}$, D. R. Nygren ${ }^{11}$, S. Odrowski ${ }^{25}$, A. Olivas ${ }^{16}$, M. Olivo ${ }^{22,15}$, A. O’Murchadha ${ }^{1}$, S. Panknin ${ }^{21}$, L. Paul ${ }^{18}$, C. Pérez de los Heros ${ }^{22}$,

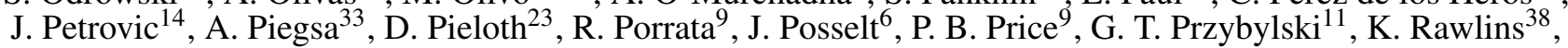
P. Redl ${ }^{16}$, E. Resconi ${ }^{25,42}$, W. Rhode ${ }^{23}$, M. Ribordy ${ }^{24}$, A. S. Richard ${ }^{30}$, M. Richman ${ }^{16}$, J. P. Rodrigues ${ }^{1}$, F. Rothmaier ${ }^{33}$, C. Rott $^{12}$, T. Ruhe ${ }^{23}$, D. Rutledge ${ }^{26}$, B. Ruzybayev ${ }^{7}$, D. Ryckbosch ${ }^{2}$, H.-G. Sander ${ }^{33}$, M. Santander ${ }^{1}$, S. Sarkar ${ }^{5}$, K. Schatto ${ }^{33}$, T. Schmidt ${ }^{16}$, A. Schönwald ${ }^{10}$, A. Schukraft ${ }^{18}$, L. Schulte ${ }^{33}$, A. Schultes ${ }^{6}$, O. Schulz ${ }^{25,43}$, M. Schunck ${ }^{18}$, D. Seckel ${ }^{7}$, B. Semburg ${ }^{6}$, S. H. Seo ${ }^{19}$, Y. Sestayo ${ }^{25}$, S. Seunarine ${ }^{39}$, A. Silvestri ${ }^{8}$, K. Singh ${ }^{20}$, A. Slipak ${ }^{26}$,

G. M. Spiczak ${ }^{3}$, C. Spiering ${ }^{10}$, M. Stamatikos ${ }^{12,40}$, T. Stanev ${ }^{7}$, T. Stezelberger ${ }^{11}$, R. G. Stokstad ${ }^{11}$, A. Stöß1 ${ }^{10}$, E. A. Strahler ${ }^{20}$, R. Ström ${ }^{22}$, M. Stüer ${ }^{21}$, G. W. Sullivan ${ }^{16}$, Q. Swillens ${ }^{14}$, H. Taavola ${ }^{22}$, I. Taboada ${ }^{28}$, A. Tamburro ${ }^{3}$,

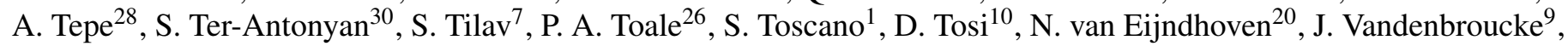
A. Van Overloop ${ }^{2}$, J. van Santen ${ }^{1}$, M. Vehring ${ }^{18}$, M. Voge ${ }^{25}$, C. Walck ${ }^{19}$, T. Waldenmaier ${ }^{36}$, M. Wallraff ${ }^{18}$, M. Walter ${ }^{10}$,

Ch. Weaver ${ }^{1}$, C. Wendt ${ }^{1}$, S. Westerhoff ${ }^{1}$, N. Whitehorn ${ }^{1}$, K. Wiebe ${ }^{33}$, C. H. Wiebusch ${ }^{18}$, D. R. Williams ${ }^{41}$,

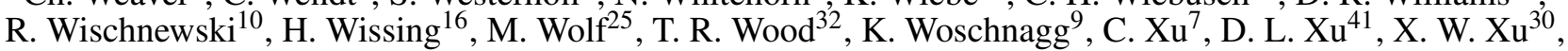
J. P. Yanez ${ }^{10}$, G. Yodh $^{8}$, S. Yoshida ${ }^{35}$, P. Zarzhitsky ${ }^{41}$, and M. Zoll ${ }^{19}$

(Affiliations can be found after the references)

A\&A 535, A109 (2011), DOI: 10.1051/0004-6361/201117810

Key words. neutrinos - supernovae: general - instrumentation: detectors - errata, addenda

This erratum corrects Fig. 16 of our original paper (Abbasi et al. 2011). The figure displays the number of standard deviation with which the IceCube neutrino observatory can distinguish between normal and inverted neutrino hierarchies, provided that the time dependent neutrino luminosities and energy spectra associated with a core collapse supernova were precisely known. We found that the abscissa of Fig. 16 was shifted to the right by $5 \mathrm{kpc}$ which led to results that were too conservative. All other conclusions of the paper remain unaffected. 


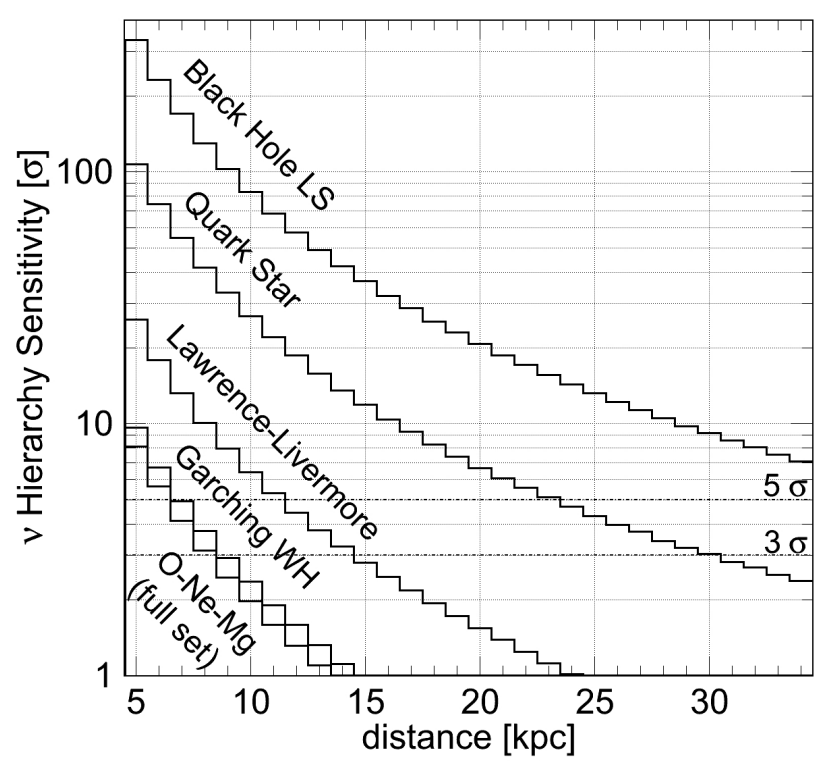

Fig. 16. Number of standard deviation with which scenarios A (normal hierarchy) and B (inverted hierarchy) can be distinguished in at least $50 \%$ of all cases as function of supernova distance for some of the models listed in Table 4. A likelihood ratio method was used assuming known model shapes.

\subsection{Neutrino hierarchy sensitivity and rate summary}

The number of standard deviation with which normal and inverted $v$ hierarchies (Scenarios A and B) can be distinguished are plotted in Fig. 16 as function of the supernova distance for selected models. The values represent the optimal cases when model shapes (but not necessarily the absolute fluxes) are perfectly known. Table 4 lists the number of neutrino induced photon hits that would be recorded by IceCube on top of the DOM noise for various supernova models. Note that the number of expected signal hits scales with $1 /$ distance $^{2}$; the dependence of the detection significance as function of distance can be read from Fig. 12.

\section{References}

Abbasi, R., Abdou, Y., Abu-Zayyad, T., et al. 2011, A\&A, 535, 109

1 Dept. of Physics, University of Wisconsin, Madison, WI 53706, USA

2 Dept. of Subatomic and Radiation Physics, University of Gent, 9000 Gent, Belgium

3 Dept. of Physics, University of Wisconsin, River Falls, WI 54022, USA

${ }^{4}$ Dept. of Physics and Astronomy, University of Canterbury, Private Bag 4800, Christchurch, New Zealand

5 Dept. of Physics, University of Oxford, 1 Keble Road, Oxford OX1 3NP, UK

6 Dept. of Physics, University of Wuppertal, 42119 Wuppertal, Germany

7 Bartol Research Institute and Department of Physics and Astronomy, University of Delaware, Newark, DE 19716, USA

8 Dept. of Physics and Astronomy, University of California, Irvine, CA 92697, USA
9 Dept. of Physics, University of California, Berkeley, CA 94720, USA

10 DESY, 15735 Zeuthen, Germany

11 Lawrence Berkeley National Laboratory, Berkeley, CA 94720, USA

12 Dept. of Physics and Center for Cosmology and Astro-Particle Physics, Ohio State University, Columbus, OH 43210, USA

13 Dept. of Astronomy, Ohio State University, Columbus, OH 43210, USA

14 Université Libre de Bruxelles, Science Faculty CP230, 1050 Brussels, Belgium

15 Fakultät für Physik \& Astronomie, Ruhr-Universität Bochum, 44780 Bochum, Germany

16 Dept. of Physics, University of Maryland, College Park, MD 20742, USA

17 Dept. of Physics and Astronomy, University of Kansas, Lawrence, KS 66045, USA

18 III. Physikalisches Institut, RWTH Aachen University, 52056 Aachen, Germany

19 Oskar Klein Centre and Dept. of Physics, Stockholm University, 10691 Stockholm, Sweden

20 Vrije Universiteit Brussel, Dienst ELEM, 1050 Brussels, Belgium

21 Physikalisches Institut, Universität Bonn, Nussallee 12, 53115 Bonn, Germany

22 Dept. of Physics and Astronomy, Uppsala University, Box 516, 75120 Uppsala, Sweden

23 Dept. of Physics, TU Dortmund University, 44221 Dortmund, Germany

${ }^{24}$ Laboratory for High Energy Physics, École Polytechnique Fédérale, 1015 Lausanne, Switzerland

25 Max-Planck-Institut für Kernphysik, 69177 Heidelberg, Germany

26 Dept. of Physics, Pennsylvania State University, University Park, PA 16802, USA

27 Dept. of Astronomy and Astrophysics, Pennsylvania State University, University Park, PA 16802, USA

28 School of Physics and Center for Relativistic Astrophysics, Georgia Institute of Technology, Atlanta, GA 30332, USA

29 CTSPS, Clark-Atlanta University, Atlanta, GA 30314, USA

30 Dept. of Physics, Southern University, Baton Rouge, LA 70813, USA

31 Dept. of Astronomy, University of Wisconsin, Madison, WI 53706, USA

32 Dept. of Physics, University of Alberta, Edmonton, Alberta, Canada T6G 2G7

33 Institute of Physics, University of Mainz, Staudinger Weg 7, 55099 Mainz, Germany

e-mail: lutz.koepke@uni-mainz.de

34 Université de Mons, 7000 Mons, Belgium

35 Dept. of Physics, Chiba University, Chiba 263-8522, Japan

36 Institut für Physik, Humboldt-Universität zu Berlin, 12489 Berlin, Germany

37 Also Sezione INFN, Dipartimento di Fisica, 70126, Bari, Italy

38 Dept. of Physics and Astronomy, University of Alaska Anchorage, 3211 Providence Dr., Anchorage, AK 99508, USA

39 Dept. of Physics, University of the West Indies, Cave Hill Campus, Bridgetown BB11000, Barbados

40 NASA Goddard Space Flight Center, Greenbelt, MD 20771, USA

41 Dept. of Physics and Astronomy, University of Alabama, Tuscaloosa, AL 35487, USA

42 Now at T.U. Munich, 85748 Garching \& Friedrich-Alexander Universität Erlangen-Nürnberg, 91058 Erlangen, Germany

43 Now at T.U. Munich, 85748 Garching

44 Now at Dept. of Physics and Astronomy, Rutgers University, Piscataway, NJ 08854, USA

45 Now at Physics Department, South Dakota School of Mines and Technology, Rapid City, SD 57701, USA

46 Los Alamos National Laboratory, Los Alamos, NM 87545, USA 